\title{
BENIGN PROSTATIC OBSTRUCTION
}

\author{
By Ronald W. ReId, M.S., F.R.C.S.
}

Surgeon, Essex County Hospital. Consulting Surgeon, Essex County Council

\section{Introduction}

The treatment of prostatic obstruction, to begin with the family doctor's responsibility, is still the business of the general surgeon despite the growth and partition of modern surgery from which urology has evolved. The separation of urology from general surgery has given impetus to the study of those disturbances of bladder function arising from pathological changes in the prostate gland, and recent years häve seen the introduction and development of many new techniques for the removal of obstructing tissue. The review of benign prostatic obstruction attempted in this paper is based largely upon personal experience, and for this and other reasons cannot in any way be complete. The multiplicity of techniques and the formidable size of the relevant literature render such a task impossible ; omissions and repetition of known facts are bound to occur, for which errors I beg the reader's indulgence.

It is important to examine the terms which have been used in the past to describe the condition known clinically as prostatic obstruction. Firstly, enlargement of the gland is not synonymous with obstruction. The majority of old men have some enlargement of the prostate but quite a small proportion of them suffer from disturbing symptoms or retention. Secondly, many of the most severe cases of obstruction have no enlargement of the gland whatever. The prostate gland lies at the neck of the bladder below the internal sphincter and surrounds the first part of the urethra. Its very position gives rise to grounds for confusion in diagnosis, and the fact that the bladder, aptly called the mirror of the urinary tract, is disturbed by practically all affections of the urinary system further adds to diagnostic difficulties. There is no doubt that benign adenomatous hypertrophy, known also as senile enlargement or adenoma of the prostate and fibrous hyperplasia or chronic fibrosis together account for the vast majority of cases of obstruction, but many other local conditions interfere with urine outflow. Among these may be mentioned contractures of the vesical orifice above the prostate which show themselves as bars and bands of hypertrophied muscle. In the prostate itself cysts, abscesses and tuberculosis may cause confusion. Stones and diverticulae in the bladder produce varying degrees of obstruction, strictures and neurogenic inco-ordination may give rise to symptoms superficially resembling those of prostatic obstruction. Whatever the essential pathology, obstruction at the bladder neck causes chronic retention of urine and deterioration of renal function.

\section{History}

In the study of human affairs history is often the key to present problems and a guide to the future, and to this rule surgery is no exception. The student is advised to glance back into the past to learn of the struggles and triumphs of his surgical forefathers before attempting to evaluate the present state of medical knowledge and probe into the future.

The symptoms of bladder disorders have been known from times immemorial, and that attempts were made by the ancients to overcome the obstruction is shown by the discovery of catheters in the equipment of Syrian and Egyptian surgeons. Anatomists of the Renaissance mention the prostate gland, but do not seem to have recognized that it became enlarged and was the cause of urinary disturbance. To Lassar, a Venetian physician who died in ${ }^{1563}$ is attributed the discovery that the bladder could be blocked by swelling of the prostate. This surgeon is said to have practised successfully an incision of the neck 
of the bladder through the perineum to relieve retention.

There is no doubt that the early history of prostatic obstruction is inseparably linked up with that of stone, and that from the Renaissance onwards itinerant stone cutters roamed throughout Europe hoisting their coloured poles in the villages which they temporarily visited. From the hands of the wandering lithotomists stone cutting passed to the qualified surgeons, and in this country William Cheseldon was one of the most able and successful. It is possible that partial prostatectomy was first performed by chance when a master stone cutter enucleated part of the prostate gland during the passage of a large stone through the forcibly dilated urethra. John Hunter, Benjamin Brodie and Chopart attempted to relieve prostatic obstruction by tunnelling the prostate with a metal catheter, and Physick of Philadelphia produced a distensable bougie which designed to dilate the narrow urethra. The first attempt to remove prostatic tissue is attributed to Mercier who devised a punch with which to tunnel the gland, but most attempts were followed by disastrous haemorrhage and infection. In I873 Bottini invented a galvanocautery which operated in a manner similar to the punch of Mercier with the additional advantage that haemorrhage was better controlled.

After the introduction of anaesthetics and antiseptic surgery the bladder could be opened from above and the prostate easily approached. By 1880 suprapubic cystotomy was recognized as a safe procedure for the relief of retention and McGill of Leeds advocated excision of parts of the gland. In the last decade of the rgth century the operation of complete enucleation of the adenomatous prostate revolutionized bladder surgery. With this radical operation two great names are associated, those of Freyer and Fuller. In Europe Freyer, whose paper was published in rgor, is looked upon as the father of modern prostatic surgery, and in America Fuller, whose account appeared in 1893 , is accorded the title. Whoever first practised the new total enucleation, there can be no doubt that the results were staggeringly good. Freyer who was a strong and courageous surgeon with the finest judgement performed over I,500 prostatectomies with a mortality of 5.3 per cent., a figure of which any present day surgeon might well be proud, but cases were carefully selected and there must 3 have been many turned away. Prostatic ${ }_{c}$ surgery was taken up by the great surgeons of the day; before long Young of Baltimore introduced his perineal approach and the suprapubic was modified by Thompson $\frac{\bar{\sigma}}{\bar{\omega}}$ Walker, Harris and many more. The last $20 \frac{\vec{\sigma}}{2}$ years has seen the development of endoscopic prostatectomy by Stern and McCarthy, ${ }^{\circ}$ Bumpus, Thompson and others, a departure? from open surgical techniques which has had $\vec{\omega}$ great influence on and may eventually revolutionize prostatic surgery.

Pathology

The pathology of prostatic obstruction can conveniently be considered under two head- $\vec{\sigma}$ ings :-

(a) The pathological changes occurring in $\stackrel{\circ}{3}$ the gland itself which cause obstruction to $?$ micturition.

(b) The effects of chronic retention and baes $\overrightarrow{0}$ pressure upon the bladder, ureters, kidneys and .N cardiovascular systems.

(a) In the great majority of cases prostatic obstruction is caused by benign adenomatous hypertrophy of the gland, a condition which is $\bar{\otimes}$ etiologically obscure despite much study on the $\stackrel{2}{\vec{P}}$ part of clinicians and pathologists. All classes $\frac{3}{3}$ of the community are affected equally and the married and celibate alike. This senile en-? largement occurs usually in men over 60 음 years of age when some growth of the gland $\stackrel{3}{-}$. normally occurs. Among the theories ad- $\frac{3}{3}$. vanced have been that the hypertrophy results from obscure involutionary trends in the endocrine system, and from changes similar to those $\circ$ which occur in the female at the menopause. The enlargement begins with small nodes of glandular hyperplasia which are separated from N the normal parent gland by capsules composed of compressed normal prostatic tissue. These ${ }_{N}$ nodes, at first small, enlarge to form irregular ${ }^{\omega}$ clumps which stretch and distort the prostatic urethra and bulge up into the bladder to form pedunculated lobes or collars surrounding the $\stackrel{\Phi}{\rightarrow}$ internal urinary meatus. Attempts have been 0 made to distinguish the various lobes of the prostate which give rise primarily to obstruc- 
tion, and attention has been drawn to the importance of the sub-cervical and sub-trigonal glands. However, little is to be gained by this detailed study of local anatomy, for in general the lateral lobes show the most marked enlargement; small adenomas may cause obstruction or a mass the size of the fist may grow before retention occurs. Whatever their size adenomatous tumours can invariably be enucleated from their false capsules with ease and satisfactory results.

In diffuse fibrous hyperplasia, a condition which is second in importance to benign adenomatous hypertrophy, there is little doubt that long standing inflammation plays an important part in etiology. The term hyperplasia may be inaccurate, suggesting as it does increase of tissue formation. It is probable that chronic prostatitis lies behind this form of enlargement and that in the early stages oedema and chronic inflammation with round cell infiltration causes the gland to enlarge, but that as time goes on the swelling subsides and scarring follows in the trail of inflammation with shrinkage of the gland. On examining such a prostate the cut surface is found to be of even texture, firm, leathery or rubbery and showing none of the spherical glandular enlargement which characterizes benign adenomatous hypertrophy. This gland cannot easily be removed whole as its sheath is the true prostatic capsule which is affected by the diffuse inflammatory change and attempts at enucleation, as would be expected, are associated with tearing of the tissues and gross surgical trauma. Microscopic examination of this gland confirms the gross appearance and in place of the spherical adenomatous globes, diffuse infiltration with leucocytes, epithelial degeneration and fibrous changes are seen. In the early stages of diffuse hyperplasia increased epithelial activity may cause prostatorrhoea, but as degeneration and fibrosis set in the gland compresses the urethra causing increasing obstruction to bladder evacuation, and in some cases inspisated secretion leads to the formation of endogenous prostatic calculi.

The chronic inflammation of diffuse fibrous hyperplasia may have its main impact upon the sub-trigonal glands which lie just inside the internal meatus under the trigone. In this inflammation the bladder mucous membrane and the trigonal muscle may take part and a $\grave{b} a r$ be formed which proves an effective obstacle to micturition. Such bars may exist with or without other changes in the prostate gland itself and may require removal as a separate procedure during the various forms of prostatectomy to be described. They can account for post-operative osbtruction unless adequately treated.

(b) Secondary changes in the urinary tract. In 1917 Young showed that at the beginning of micturition the trigone is depressed by contraction of the trigonal muscle which pulls the median and posterior parts of the prostate downwards to open the internal urinary meatus. Contraction of the general bladder musculature follows and the urine is expelled. This movement of the trigone can be easily confirmed by cystoscopy. By whatever method the prostate obstructs the bladder, whether it is by elongating and distorting the urethra or by compressing it, the onus of opening the internal sphincter rests with the trigonal muscle and so one of the earliest signs of prostatic obstruction is hypertrophy of this muscle, a change soon followed by genera bladder hypertrophy. The bladder musculature is normally an even sheet of crossing muscle bands completely supporting the mucous membrane, but in obstruction the intravesical pressure forces pouches of mucous membrane through the hypertrophied bands to form saccules or diverticula; this appearance is characteristic of a bladder obstructed at its outlet. The ureters which, by peristaltic action, inject urine into the bladder at frequent intervals are protected by a valvular muscular mechanism at their orifices, sufficient for a considerable time to protect the upper urinary tract from increased intravesical pressure, and there is little doubt that this mechanism remains effective for a very long time. However, as the bladder distends the ureteric orifices lose their efficiency and no longer protected, the kidneys must secrete against an increased load with results to be discussed later. There are gounds for believing that the effects of prostatic obstruction on the kidneys are not always entirely due to incompetence of the ureteral sphincters, but that the enlarging gland itself may actually compress or distort 
the lower ends of the ureters thus giving rise to obstruction at the ureteric orifices as well as at the vesical outlet. That such obstruction occurs in carcinoma is well known but it is not generally realized that large simple glands may do the same thing. Many surgeons have noted that bladder drainage fails occasionally to relieve renal back pressure and that prostatectomy is a more satisfactory primary procedure. This paradox can be explained by considering the influence of prostatic enlargement on the trigone as a whole.

When pressure in the upper urinary tract rises the kidneys secrete against a resistance and the tubules are the first structures to suffer, causing inefficient concentration of urine and polyuria. To combat increasing resistance, filtration pressure must rise and this in turn reflects upon the cardiovascular system causing hypertension and cardiac hypertrophy. The old clinicians noted the association of the enlarged prostate and the enlarged heart. As time goes on renal function deteriorates, uraemia develops and is followed by cardiac failure with attacks of nocturnal orthopnoea and pulmonary congestion.

At any stage of prostatic obstruction infection may reach the bladder through the urethra or by the blood stream, and the organisms multiply in the stagnant urine and spread like wild fire to the kidneys, where the destructive effects of pressure are increased ten-fold. Hence the importance of avoiding careless instrumentation. Infection once established leads to rapid deterioration of general health and kidney function, encourages the formation of stones and adds greatly to the gravity of the situation.

\section{Symptomatology}

The symptoms of prostatic obstruction are well known and consist of gradually increasing difficulty and frequency of micturition, of a thin or halting stream and attacks of acute retention of urine. They vary in their severity from time to time in the same case; they are often thought by the patient to be natural to old age. Occasionally transient haematuria presents or the chronic ill health and anaemia of failing kidneys, and not bladder trouble, sends the man to his doctor. As obstruction of the urine outflow develops so do the muscles of the bladder hypertrophy in response to increased work. At first the hypertrophy is sufficient to overcome the obstruction and apart from a little delay or urgency there may be few symptoms, but against increasing obstruction the compensation fails, residual urine makes its appearance and the bladder undergoes progressive and silent dilatation. Frequency then becomes conspicuous and as back pressure makes its mark upon renal function a more dilute urine is secreted and polyuria by day and night further adds to the frequency. As obstruction and renal failure increase the patient's nights are more disturbed, eneuresis may develop, thirst increase and lack of sleep and uraemia bring the patient to a sorry state. Should severe infection supervene, intolerable frequency, stranguary, haematuria and bladder irritability are followed by chills, pain in the loins and rapid physical deterioration. If the patient survives the infection without acute retention he develops some immunity, but stones may grow and infection flares up occasionally in attacks of epididymitis.

\section{Physical Signs}

Examination of a case of suspected prostatic obstruction begins with a general survey of the patient. Hard and tortuous temple arteries, baldness or deafness and the arcus senilis suggest that the patient is older than his years. Drowsiness, a dry tongue and heavy breathing are characteristic of the uraemic state. Breathlessness and oedema of the ankles may indicate cardiovascular deficiency and call for an investigation of the heart, lungs and the blood pressure and for the advice of a general physician before treatment is planned. The pupillary reactions and tendon reflexes should be checked and the external genitalia and testes inspected. On examining the abdomen a distended bladder may be found, which if present immediately after micturition indicates gross retention. If the patient is not too fat or muscular, pressure upon the apparently empty bladder may cause the desire to pass water, and if urinary infection is present the bladder and the kidneys may be tender. To examine the prostate per rectum the knee elbow position is very satisfactory ; the gland may also be palpated bimanually 
with the patient on his side, the knees up and the abdominal muscles well relaxed. It is important to recall that the prostate gland is pushed down and rotated by the distended bladder and its size is thereby exaggerated as judged by rectal examination. For true assessment the bladder must be empty. Most surgeons have their own size indexes for the prostate gland; it has been my practice to record a gland twice the normal size as being plus one and an enormous prostate of great depth and width and bulging far back into the rectum as being plus four. The size, contours, consistency and fixity of the prostate and the state of the vesicles should be noted and recorded by drawing or on a special chart.

\section{Special Examinations}

Before a plan is made for the treatment of a case of recognized or suspected prostatic obstruction the surgeon requires all the evidence he can muster. Firstly an X-ray should be taken of the whole urinary tract to discover whether the case is complicated by stones, or unsuspected carcinoma has thrown down secondary deposits in bone. Secondly, since bladder obstruction sooner or later damages the kidneys special tests of renal function must be carried out to supplement clinical examination. The first sign of incipient renal failure is a persistently low specific gravity of the urine and nocturia. The damaged kidneys cannot concentrate neither can they perform their excretory function mainly by day. A persistently low specific gravity of the urine and the passage of large volumes by night indicate that failure has set in, but the gravity test upon which Freyer placed so much confidence, is time-consuming and has been supplanted by the estimation of renal function by other means. In my opinion the most reliable and convenient test is the urea content of the blood which normally is less than $\mathbf{4 5}$ milligrammes per cent. This test combined with a sound clinical assessment of the case provides all the evidence the surgeon requires to make his decision, and more complicated investigations are time-consuming both for nursing staff and the laboratory. In ordinary circumstances they need not be pursued but they are doubtless of value in the border-line cases.
A complete examination of the urine should be carried out including culture for bacteria. The presence of albumen, blood, casts or pus indicate that the urinary tract is suffering from damage other than that due to simple obstruction. The bacterial content of the urine gives valuable information on the management of the case, for heavy infection may require a period of preliminary bladder drainage before radical surgery can be undertaken sith safety. Information will also be gained as to the therapeutic cover necessary during the operation and post-operative period.

It is the custom of many surgeons to pass a catheter and measure the residual urine, for it has been authoritatively stated that the presence of $4 \mathrm{oz}$. or more is an absolute indication for surgical treatment. The presence of residual urine is no single guide or absolute indication for surgery unless it is large in amount, obvious on abdominal examination and associated with severe symptoms and sound evidence that renal function is suffering. In the aged and infirm a considerable amount of residual urine is compatible with comfort and safety, and the patient will live longer if he is not subjected to instrumentation and is treated conservatively. However, the amount of residual urine gives important information and should be determined in every case were it not for the risks of catheterization. It has long been known that to pass a catheter on an obstructed bladder without the most aseptic precautions, such as those obtaining in the operating theatre, is dangerous, for the bladder is easily infected with organisms carried up from the pendulous urethra and the consequences of ascending urinary infection are disastrous and unpredictable. To the importance of asepsis in every stage of prostatic surgery the attention of surgeons has once more been called by Wilson Hey.

Cystoscopy provides valuable information upon the exact anatomy of prostatic obstruction and by its use general bladder or trigonal hypertrophy can be seen, and the presence or absence of diverticula, growth or stones determined. For this purpose the direct vision cystoscope is probably the most convenient as it gives an excellent view of the prostatic urethra and the bladder neck. However, as with catheterization, cystoscopy is not free 
from danger and should on no account be adopted as an out-patient procedure, but should be reserved for the moment before operation.

\section{Differential Diagnosis}

The differential diagnosis of prostatic obstruction resolves itself into the consideration of causes other than those within the prostate which may interfere with the bladder function and urine outflow, and the examination of the exact nature of the prostatic pathology. Frequency and polyuria arise from general diseases, especially those affecting the circulatory system and kidneys. Tabes dorsalis and other affections of the central nervous system interfere with bladder function and cause chronic retention; cystitis, tuberculosis, stone and bladder new growths may produce symptoms easily confused with those of prostatic obstruction, and in the urethra strictures may simulate and accompany prostatic enlargement. By a careful attention to history, physical examination, X-ray and laboratory investigations, the differential diagnosis can be narrowed down to prostatic abnormalities. The exact nature of the pathological change in the gland cannot always be determined until microscopy after operation, but generally speaking the finger in the rectum will solve the problem. Benign adenomatous hypertrophy gives rise to rounded elastic swellings which move in a mass freely within the pelvis. Diffuse fibrous hyperplasia causes some enlargement of the gland, in which case it is leathery, broad and flat, or slightly bulging and later on as fibrosis supervenes it is contracted and hard. It is always movable in the pelvic cavity, harder than benign hypertrophy but not stony and fixed as in carcinoma. Calculi may be felt in the fibrous gland and may be confused with the somewhat similar nodularity of tuberculosis or carcinoma. Tuberculosis of the prostate is invariably associated with tuberculosis of the vesicles and very often disease in the testicles and kidneys, and when suspected a general survey of the urinary system should be undertaken and examination made of the urine for tubercle bacilli. Carcinoma may arise in a previously normal or abnormal prostate and characteristically feels like a stony plaque which obscures the median groove and $\stackrel{\text { Oै }}{*}$ upper margin of the gland, extends later to the pelvic cellular tissue to fix it and finally spreads 3 round the rectum along the pelvic floor to $\frac{\varnothing}{2}$ deposit secondaries in bone. Confirmatory changes in the serum acid phosphatase level may be found.

To sum up the main points in the investigation of a case of prostatic obstruction, most in- $\frac{}{\vec{D}}$ formation can be gained by general clinical $\stackrel{\Phi}{2}$ examination, $\mathrm{X}$-rays are important for dis- $\infty$ covery of stone or secondary deposits from $\vec{\circ}$ malignant disease, the blood urea test is the simplest and most reliable index of renal $\omega_{\stackrel{\omega}{~}}$ damage and detailed examination of the urine $\frac{8}{\square}$ is essential before the plan of treatment can be 3 . carried out. More complex investigations such as intravenous pyelography, urea clear- î ance tests and cystoscopy apart from an immediate pre-operative procedure, though $\vec{\sigma}$ essential in the difficult case, are, in my o experience, unnecessary as a routine if clinical study is thorough.

\section{Complications}

The complications of prostatic obstructiog are many, affecting not only the genito-urina tract but the cardiac and pulmonary systems well, and they include many of those conditions which may properly be called the diseases of old age. Commonly encountered are :-

(r) Acute retention of urine.

(2) Cystitis and ascending renal infections.

(3) Diverticulum.

(4) Bladder stone.

(5) Epididymo-orchitis.

(6) Uraemia.

\section{Acute Retention}

Acute retention of urine, precipitated by ex- $\frac{D}{2}$ posure to cold, over indulgence in drink, infection or any acute illness such as fracture or $\%$ pneumonia, is sometimes the first symptom of $\mathrm{O}^{\circ}$ prostatic obstruction in which case the gland is $\underset{\omega}{\mathrm{W}}$ often large and soft and prone to congestion, $\stackrel{\square}{2}$ and the kidneys unaffected. In other cases, $\stackrel{\square}{\subseteq}$ the acute attack follows long standing chronic ${ }_{\infty}^{\Phi}$ retention with advanced renal damage. The two conditions should be distinguished for the first variety often responds to simple treatment 
such as rest, morphia and hot baths and in the last resort the passage of a catheter with full asepsis, while the second type demands the most careful decompression to avoid precipitating renal haemorrhage and irreversible failure. If a catheter is used, careful asepsis, good local anaesthesia, generous lubrication and extreme gentleness must be employed: The best instrument is the Foley self-retaining catheter which, having a distensible bag at its tip, requires no penile strapping to keep it in. Thus secretion drains freely out provided the catheter is smaller than the urethral calibre and urethritis and epididymitis are avoided. It is wiser also to leave a catheter in situ rather than employ repeated passage which may carry up infection. It can be removed when the general lines of treatment have been settled. Should a soft rubber catheter not pass, a bicoudé gum elastic may be gently tried, but silver instruments should be eschewed. Damage to the urethra or prostate by rough instrumentation prejudices the patient's chances of safe recovery.

If catheterization is impossible or dangerous, suprapubic cystotomy or aspiration is called for. The latter procedure, though less satisfactory than cystotomy is simple, but in my experience neither satisfactory nor safe.

\section{(2) Cystitis}

Infection may reach the obstructed bladder from the blood stream or by instrumentation. In its severe form, fortunately uncommon, symptoms are always aggravated, acute retention may supervene, the invasion may spread to the kidneys giving rise to chills, fever and septic pyelonephritis with all its disastrous consequences. Severe infection with obstruction is an absolute indication for free drainage of the bladder, forced fluids and properly chosen chemotherapy. As prolonged drainage is usually necessary cystotomy is more satisfactory than the indwelling catheter which may cause an aggravating urethritis and epididymitis.

\section{(3) Diverticulum}

Diverticula of the bladder, so easily overlooked, are large sacs unsupported by vesical muscle, and are not to be confused with the small sacs pushed into the bladder wall by increased intravesical pressure. The latter are of little significance and disappear when obstruction is relieved, but the former, although they owe their growth to bladder obstruction, do not diminish in size when the outflow is cleared but remain as cul de sacs which will harbour great quantities of urine and foster infection. Prostatic obstruction complicated by diverticulum cannot be cured by prostatectomy alone, and in suitable patients the diverticulum should be removed first and the bladder drained suprapubically for a week or two before an attack is made upon the prostate through the suprapubic wound or by other methods. If prostatectomy is done in the presence of a large diverticulum, bladder sepsis is certain to be troublesome and the subsequent diverticulectomy made more difficult by scar tissue.

\section{(4) Bladder Stone}

Stone and prostatic obstruction have long been confused. Long standing obstruction, especially with infection, favours the formation of stones which do not always give rise to the classical symptoms of stranguary, stammering bladder and painful stoppages. For this reason all cases should be examined by X-ray, including in the field the kidneys and bladder. Large or multiple stones should undoubtedly be removed by suprapubic cystotomy, and if other conditions allow the prostate may be enucleated at the same time. Small stones, and those lying above the fibrous type of prostate can be crushed and removed with Bigelow's evacuator, the obstruction being treated at the same time by endoscopic resection.

\section{(5) Epididymo-orchitis}

This complication occurs more often after prostatectomy than before, and most surgeons advise pre-operative division of the vas as a prophylactic measure. There seems little doubt that infection spreads along the vas from the urethra or prostatic bed. Before operation this complication occurs if an indwelling catheter is so applied as to interfere with the natural drainage of secretions caused by the catheter foreign body. Too large a catheter, rough work and subsequent oedema, tight straps and caked dressings on the glans 
penis, all interfere with urethral drainage and predispose to ascending infection. Chemotherapy, attention to asepsis and to catheter fitting with adequate support of the testes by wadding, have in my experience eliminated this complication; so much so that tying the vasa has been abandoned for 5 years. Epididymo-orchitis is uncommon after the transurethral operation, possibly due to the fact that little damage is done to the prostatic bed and the patient is never confined to bed for more than a few days.

\section{(6) Uraemia}

It is a remarkable fact that the kidneys can withstand the effects of back pressure for years without gross damage provided that, among other things, the ureteric orifices are neither obstructed nor incompetent, and that from severe impairment complete recovery is possible. Unrelieved obstruction in time leads to renal failure and uraemia, a metabolic disaster associated with, but not caused by, a rise in the urea content of the blood. The treatment of uraemia, the clinical picture of which is well known, consists of the relief of the causative obstruction and of measures to increase the output of urine.

Obstruction must be relieved gradually if it is of long standing and the renal function depressed. It is usual to drain the bladder by catheter or suprapubic tube and to attach to the outlet some mechanism such as a transfusion drip feed and clip which will control the rate of decompression. Haemorrhage indicates too much haste and generally 12 to 24 hours must elapse before the urine is let run free.

Unless the kidneys are irrevocably damaged diuresis will follow decompression provided the blood pressure is maintained. This is a most important factor for the kidneys cannot secrete unless the blood pressure is sufficient to maintain a vigorous glomerular circulation. Shock and debility should be treated by plasma or blood transfusion if need be until the estimated normal blood pressure is restored. At the same time the patient must drink very freely and nurses must encourage or bully him, especially if he is drowsy. Morphia and other sedatives should be avoided lest the patient's drowsiness is increased, his drinking aban- doned and his blood pressure lowered, all of which put him on the path to renal failure. Intravenous fluids, particularly saline, should 3 be used with the greatest care for in the sick $\stackrel{\varnothing}{\circ}$ and aged the pulmonary circulation is easily $\stackrel{c}{\Rightarrow}$ overloaded with subsequent oedema of the $\overrightarrow{\vec{s}}$ lungs. Oedema of the lungs induces anoxia which, in turn, causes more oedema and $\bar{E}$ anoxia of the heart muscle and the renal $\frac{\bar{p}}{\overrightarrow{0}}$ cortex. In the presence of a failing heart and $\propto$ dropping blood pressure, intravenous fluids $\%$ can easily seal the patient's fate and should be $\vec{\circ}$ used only in the presence of definite signs of $\overrightarrow{ }$ dehydration and when other methods of taking $\vec{\sigma}_{\mathscr{\sigma}}$ fluids fail.

\section{Treatment}

The treatment of prostatic obstruction cannot be bound by hard and fast rules; its $\vec{\sigma}$ aim is the saving of life and restoration of normal micturition by removal of obstructing tissue; it depends upon three main con- $\vec{D}$ siderations :-

(I) Obstruction is caused by many different $\overrightarrow{0}$ pathological changes in or near the prostae gland, for the treatment of which have beef devised many operations, none suitable fơr every case. From the point of view of the technical problems involved, the causes of $\stackrel{0}{\circ}$ simple obstruction fall naturally into two $\stackrel{\varnothing}{\varrho}$ groups. Benign adenomatous hypertrophy, $\overrightarrow{\overrightarrow{0}}$ the big group, different from all others in that 3 the obstructing tissue can be cleanly enucleated from its false capsule with satisfactory results. On the contrary, diffuse fibrous hyperplasia and the common forms of bar cannot be so $\dot{0}$. enucleated and must be resected either by open operation or through the urethra. Enucleation 8 is one of the simplest techniques in operative surgery, transurethral resection one of the 9 more difficult.

(2) Prostatic obstruction generally affects the aged whose general physique is fit to $ᄋ$ withstand neither the strain of a long or severe $N$ operation whatever its intrinsic technical $\stackrel{N}{\omega}$ merits, nor protracted illness and recumbency.. Be it allowed that among the cases are many ${ }^{\circ}$ middle aged and fit men, the fact remains that they do not provide the real problem. An operation suitable for them may be quite unsafe for the aged ; conversely, what is safe for 


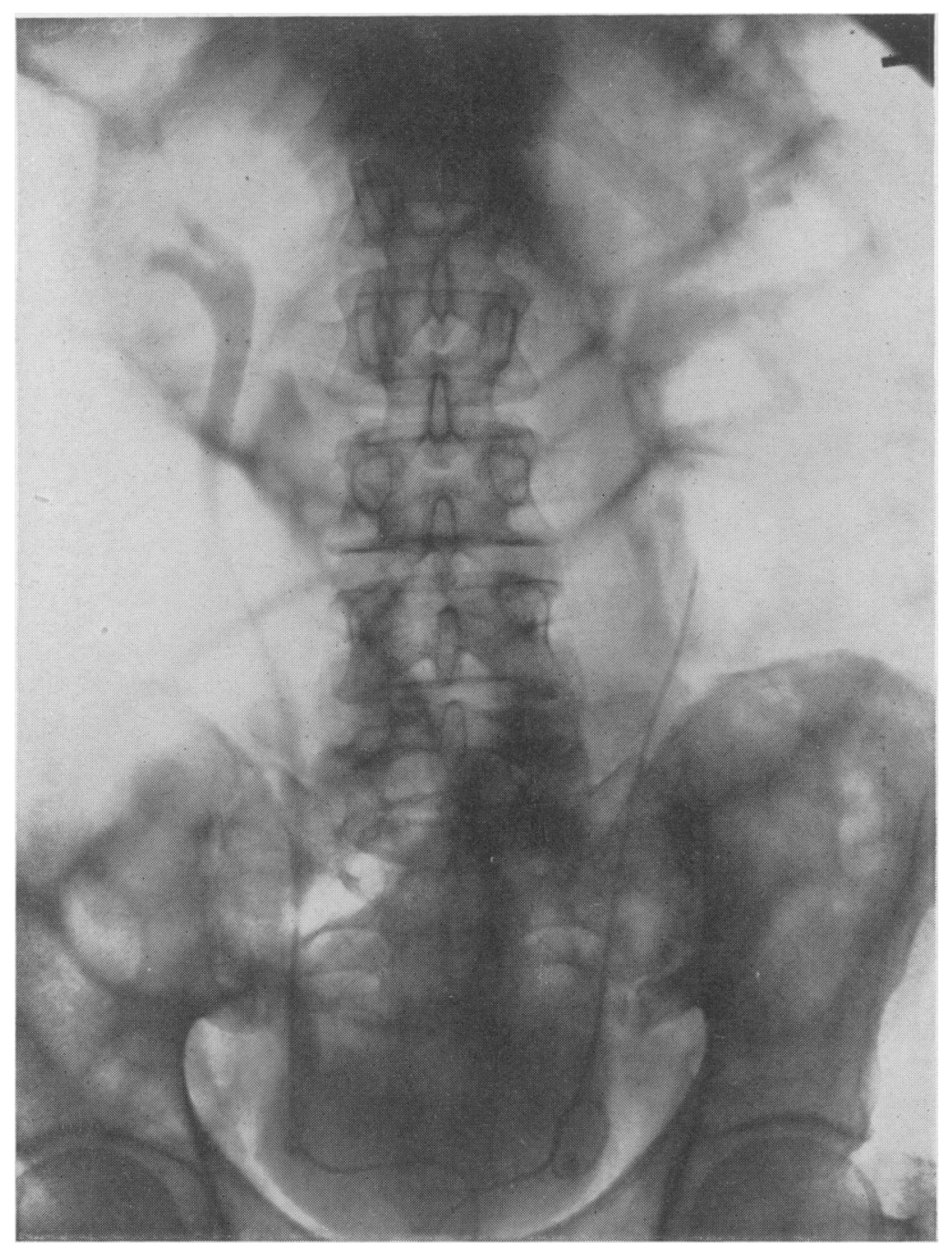

Ascending pyelogram showing dilatation of upper urinary tract due to enlarged prostate. In this case there is no obstruction of the ureter. 


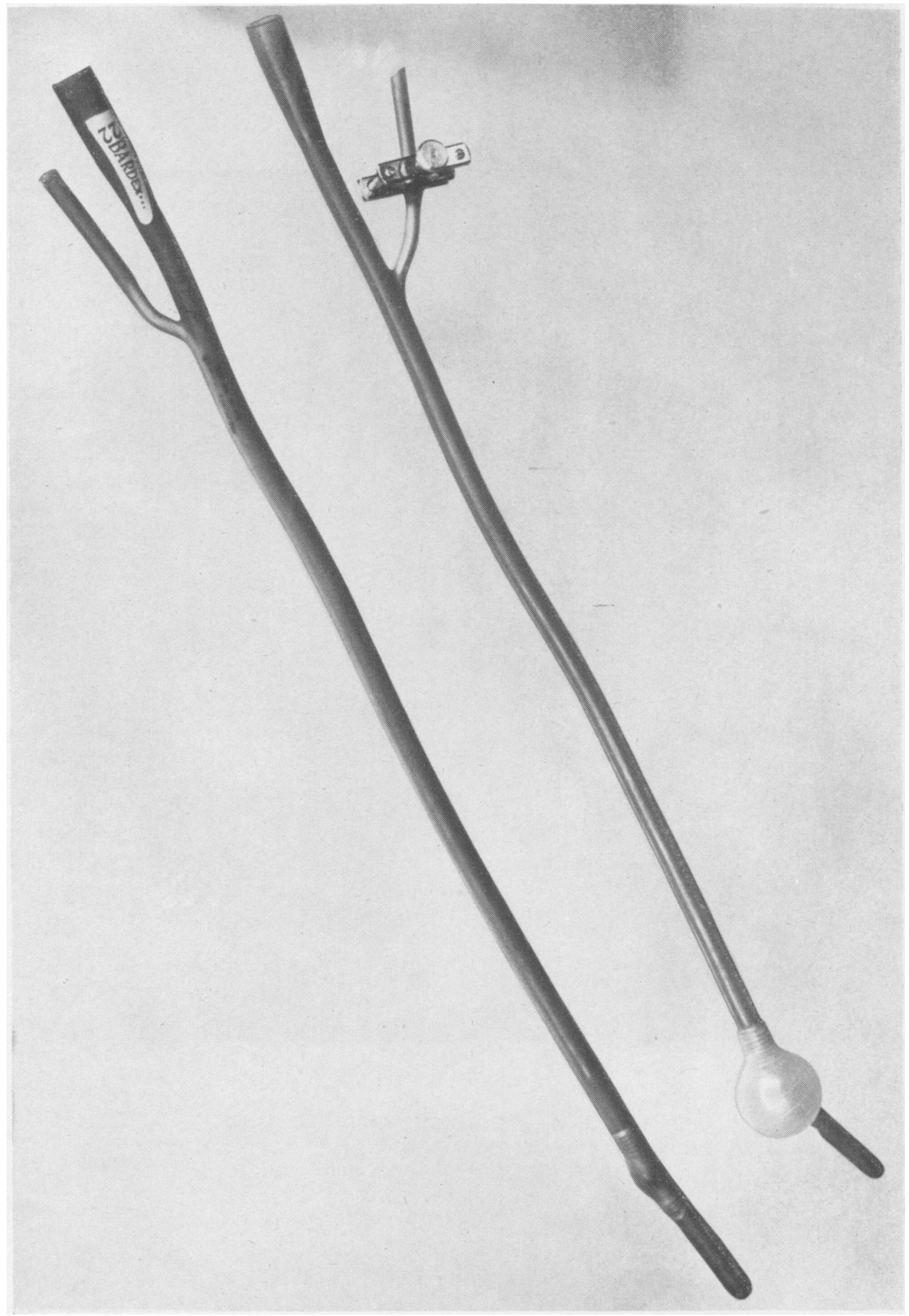




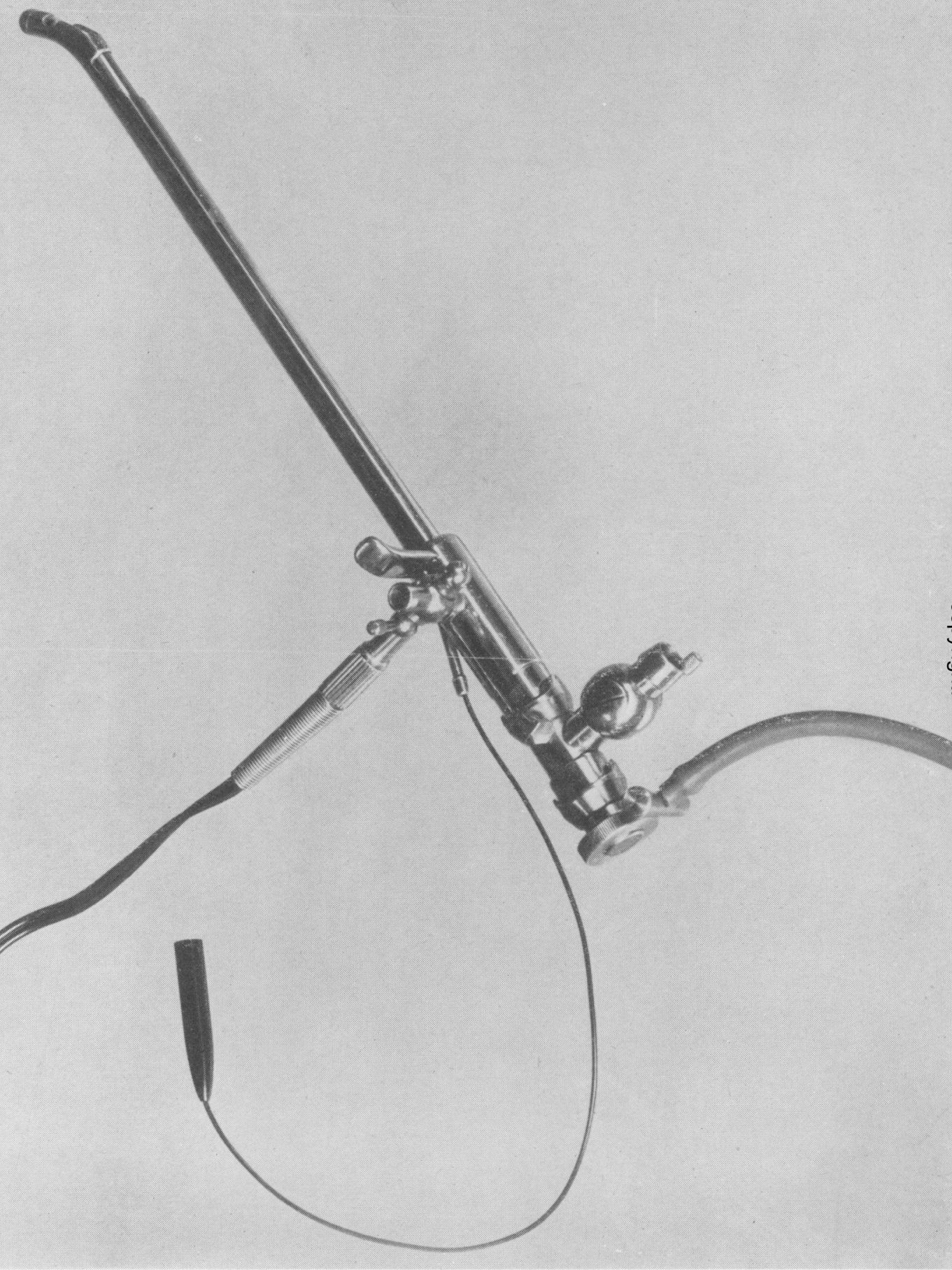

윰 \. .

o

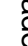




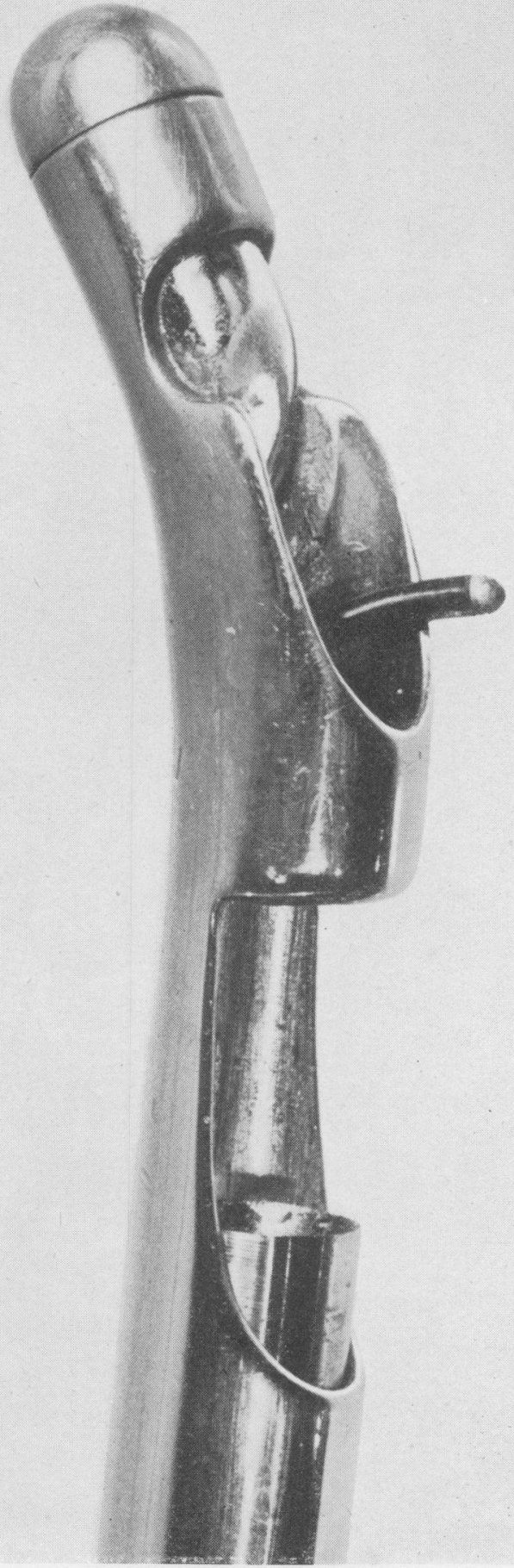


the aged must be safer still for the middle aged provided always that the method gives satisfactory results.

(3) In many instances the patient is worn out, the urinary tract infected, the kidneys damaged or the cardiovascular and pulmonary systems embarrassed. The surgeon must take account of these and other factors in planning treatment and the advice of a physician should be freely taken. Treatment on general lines and prophylaxis for complications should be carried on throughout for the operation is but a step, important but not paramount in the scheme.

\section{Preliminary Drainage}

Since prostatic obstruction causes retention of urine, in some cases complete, in others partial, the question of preliminary drainage is of importance. Acute retention must be relieved unless the patient can be transferred to hospital and have his prostate removed within a few hours. Chronic retention is often associated with sepsis and renal failure or other complications rendering immediate prostatectomy dangerous, and the only hope of relief and improvement lies in adequate drainage. Drainage relieves back pressure upon embarrassed kidneys and allows free exit of infected urine but at the same time the tube introduces infection into the bladder which may be sterile so far. Despite Wilson Hey's advocacy of primary prostatectomy for acute retention regardless of complications, most surgeons would agree that there are certain definite indications for simple drainage as follows :-

(a) Acute retention.

(b) Gross bladder sepsis.

(c) Advanced renal failure.

(d) Extreme feebleness of the patient and cardiovascular degeneration.

Drainage of the bladder can be effected either by indwelling catheter or suprapubic cystotomy. The indwelling catheter is simple in use, drains the bladder from its lowest point and, provided it is of small size and passed and fixed without damage to the urethra, it can be retained for 2 or 3 weeks. The Foley type catheter has many great advantages. Changing the catheter does nothing but carry up into the bladder infected secretion from the pendulous urethra. Suprapubic cystotomy has the great advantage that drainage can be maintained for an indefinite time and unless there are sound reasons for opening the bladder freely, the tube should be inserted under a local anaesthetic by the trocar and cannula such as that devised by Riches. By this means active surgery is kept to the minimum-an advantage in the aged. The tube should be inserted high in the bladder, taking care to avoid the peritoneum, in order that the oblique high stoma may subsequently close easily. There is nothing more trying to shut than a fibrous fistula situated upon the symphysis pubis.

In the absence of complications there is nothing to be gained by preliminary drainage of any kind and the sooner a direct attack is made upon the obstruction the better for the patient. With complications, efficient drainage combined with hydrotherapy and suitable antiseptics diminish infection and prostatic congestion, and improve renal function, thus making the subsequent major operation safer.

\section{Operative Treatment}

The operations commonly employed for the relief of prostatic obstruction are listed and grouped below. The steps of many of these operations are well known, and no attempt will be made to give details of technique ; only the broad outline can be described.

\section{A. Suprapubic Cystotomy.}

B. Transvesical Prostatectomy.

(I) Simple enucleation with open drainage. (Freyer-Fuller operation.)

(2) Simple enucleation with open haemostasis and drainage. (Thompson Walker operation.)

(3) Simple enucleation with restoration of trigone, closure of bladder and catheter drainage. (Harris operation.)

(4) Simple enucleation with open haemostasis, closure of the bladder and catheter drainage. (Wilson Hey operation.)

C. Extravesical Prostatectomy.

(I) Perineal prostatectomy. (Young's operation.) 
(2) Retropubic prostatectomy. (Millin's operation.)

D. Transurethral Prostatectomy.

(I) Electro-resection of Stern, McCarthy, Kirwin and others.

(2) Cold punch of Braasch, Bumpus, Thompson, Emmett and others.

\section{Suprapubic Cystotomy}

Suprapubic cystotomy unless performed as a temporary measure is a confession of failure. Unfortunately many old and feeble patients are fit for nothing more than this simple operation which, despite the simplicity, carries a high mortality. In my series the mortality is in the neighbourhood of 30 per cent., which is evidence of the extreme debility of rejected cases. It is the common experience of most surgeons, however, that many cases suffering from advanced failure including gross oedema and ascites may, in the course of time, recover sufficiently to warrant a more radical operation. It is surprising how prolonged bladder drainage reduces the size of the soft prostate and how easily normal micturition can be restored in what were once impossible cases by a small transurethral resection. The steps of suprapubic cystotomy are well known but I would stress the importance of doing the operation under local anaesthesia and using the simplest technique possible.

\section{Transvesical Prostatectomy}

Simple enucleation of the prostate with open drainage is one of the most satisfactory operations in surgery, for by it all the obstructing tissue is removed, haemorrhage can easily be controlled by packing or haemostatic bags and closure of the bladder wound usually takes place within 20 days. The operation can be performed by anyone with average surgical knowledge and skill, but it is often associated with considerable shock, with reactionary haemorrhage and bladder sepsis, with several days complete recumbency and many weeks invalidism. Simple though it is to perform, this operation is severe, and in average hands carries a mortality of 10 or more per cent. If the rejects and the failures are taken into consideration the results cannot be looked upon with complacency. The more careful control of haemorrhage advocated by Thompson Walker gives better results, but the operation is more prolonged and severe and greater surgical skill is required for its performance. Thompson Walker with his great experience and care carried out this operation with brilliant results. Ten years ago Harris introduced his operation in which haemorrhage was controlled by ligature and suture and the prostatic bed was covered with flaps of vesical mucosa . and the bladder closed. This ideal operation in Harris's hands produced good results, but it is a severe procedure for the aged requiring a long anaesthetic, and suturing the raw area interferes with drainage and may cause spreading pelvic cellulitis and postoperative obstruction due to scar tissue. Failure to secure complete haemastasis has caused many surgeons to modify this operation by inserting a fine suprapubic tube, a safety measure which does not detract from the value of the operation. Wilson Hey of Manchester has practised primary prostatectomy in all cases despite complete retention or a high blood urea and his results are most impressive. He stresses the importance of a most rigid aseptic technique, and having removed the prostate and controlled haemorrhage with theo free use of the diathermy, he passes a catheter retrograde from the bladder to the penile urethra, thus avoiding pushing up organisms from the pendulous urethra, when the catheter is inserted in the usual manner. At the end of the operation the bladder is closed, filled with citrate and connected to a closed drainage system. I have not been able to achieve the degree of haemastasis required for primary bladder closure without the very heavy use of the diathermy, but my limited experience of this operation is so far satisfactory. Sepsis and sloughing have not occurred nor has there been bleeding with returning blood pressure. There seems to me no reason why this operation could not be made safer by the insertion of a fine suprapubic tube as in the modified Harris operation, nor would it seem that much is gained by the somewhat tedious insertion of the catheter in retrograde direction.

\section{Extravesical Prostatectomy}

In cxtravesical prostatectomy the gland can be approached from behind through the 
perineum, or from in front across the cave of Retzius. In the early part of the century, Hugh Hampden Young invented his perineal operation by which can be removed not only adenomatous tumours but fibrous glands and carcinoma. The operation has modifications, but basically consists of an approach to the posterior surface of the gland through the perineum, incision of the capsule and removal of all obstructing tissue. Haemorrhage is controlled under direct vision, stones can be removed from the bladder and the normal anatomical arrangements are restored by suture after a tube has been inserted into the bladder. The perineal operation has given brilliant results in the hands of Young and his followers, but it has never been really popular in this country. My experience of this operation is too limited for comments to be of value, as the few cases in which this operation was employed were excellent surgical risks and could have been cured by almost any method, there were no deaths, and the two complications incontinence and fistula were not encountered. It is my impression that the operation cannot be carried out in the aged with impunity.

Retropubic prostatectomy. It is no exaggeration to say that in 1945 the urological world was startled by the introduction of retropubic prostatectomy. Millin, whose experience of prostatic surgery in England is unrivalled, did not accept the limitations implied by the known methods of prostatectomy and devised his own approach. Briefly Millin's operation consists of exposing the anterior surface of the prostate through the cave of Retzius. Haemorrhage, chiefly from the dorsal penile vein, having been checked by diathermy or ligature, the true and false capsules are incised transversely and a line of cleavage sought. The adenomatous masses are separated by blunt or scissor dissection in a downward direction until the urethra at the apex of the prostate is cleared all round. This structure is severed and the mass turned upwards and dissected free until it is attached to the bladder solely by a cuff of mucous membrane, which is then divided and the tumour removed. The opening into the bladder is enlarged by excision of a wedge from the truncated cuff to avoid post-operative obstruction by a diaphragm and a catheter is passed" up the urethra into the bladder. Haemostasis is secured by ample suture of the prostatic capsule and the abdomen is closed with simple drainage of the retropubic space. The catheter is attached to a closed drainage system and is removed in 3 to 7 days. This operation gives good results, haemorrhage from the bladder is uncommon, normal micturition is soon re-established and the wound heals by first intention. Every operation has its disadvantages and in the case of Millin's they are, in my experience, as follows :-

(a) In fat men the approach to the anterior surface of the prostate is through a deep dark hole, haemorrhage can be troublesome before the capsule is incised, and unless the operator possesses considerable experience and special equipment, the operation takes time, requires deep anaesthesia and causes shock.

(b) Unless dissection is light and careful, or if rough enucleation be substituted for such care, quite severe shock develops particularly in the aged patient. In my experience this shock is more severe than would be expected in a similar prostatectomy by the FreyerFuller technique.

(c) Reactionary haemorrhage may cause a pelvic haematoma, which, if infected goes on to pelvic cellulitis.

(d) The fibrous prostate cannot readily be separated from the capsule without severe trauma.

(e) Unless the vesical opening be enlarged, a diaphragm may develop, cause postprostatectomy obstruction and require resection. This occurred in two out of my first seven cases.

(f) The catheter may slip out and be difficult to replace.

Lest it be thought that the disadvantages weigh heavily, retropubic prostatectomy is, in my opinion, a great advance in prostatic surgery. It is not so simple as transvesical enucleation, it is not yet an operation for the occasional prostatectomist, but in the hands of Millin it gives excellent results.

\section{Transurethral Prostatectomy}

Transurethral resection is the oldest operation devised for the relief of prostatic obstruction by removing tissue, for more than 100 
years ago: Mercier invented his punch, and many similar instruments have since been introduced. Hugh Young and Kenneth Walker used their punches 30 years ago, but modern resection is an operation of the last 15. years' and is associated with the names of Stern and McCarthy, of Bumpus, Braasch, Thompson, Emmett and others. There is little doubt that the modern operation is largely American in origin and development.

In the Stern-McCarthy operation prostatic tissue is removed by the diathermy loop which effects haemostasis as it cuts. The method is very useful for small fibrous glands and for carcinoma but it is difficult to remove much tissue and, in my limited experience, not easy to be sure where the cuts are being made. However, the Stern-McCarthy method is widely used in Europe and America and in skilled hands and suitable cases the results are good.

The cold punch resectoscope, a modification of the direct vision cysto-urethroscope, contains a sliding circular knife which cuts away parts of prostatic tissue engaged in a terminal fenestra. The pieces are washed into the bladder by a continuous stream of lotion and withdrawn by suction.

With the Gershom-Thompson resectoscope, the instrument with which I am most familiar, larger amounts of prostatic tissue can be removed at one. sitting. The operation is carried out under low spinal anaesthesia, the resectoscope which is of large calibre is passed with the greatest care as the urethra is easily damaged, an accident : which invariably produces troublesome strictures, the inside of the bladder, the internal urinary meatus and the prostatic urethra inspected and a decision made as to whether the case is one suitable for the method. Large adenomatous prostates weighing over $100 \mathrm{gm}$. are extremely difficult to resect, but as the operator becomes more experienced so will he be able to take more tissue including the difficult lateral and intravesical lobes. The removal of bars and fibrous glands is relatively easy and a sufficiency of tissue can be simply removed from a carcinoma.

Resection starts with removal of part of the median lobe and then each lateral lobe is taken separately and haemostasis secured before passing on to the opposite side. The cutting may proceed briskly until the apex of the gland is reached where care must be taken lest the external sphincter be damaged. To cut below the verumontanum is disastrous. As the operation is completed the fibrous capsule should be seen all round except in front, and bleeding points should be controlled with the diathermy electrode. Provided resection is clean and haemostasis careful and, provided also, the patient's blood pressure has not dropped unduly, the resectoscope is withdrawn with the bladder full. Pressure upon the pubis should produce a good urinary stream if results are to be satisfactory. A Foley catheter is then inserted and the bag is distended with 20 ccs. of lotion and drawn down into the prostatic cavity. Careful adjustment of the bag and variations in the amount of fluid in it are required to control bleeding completely. At the end of the operation the patient is returned to the ward where bladder washes are usually continued at frequent intervals to avoid clotting. This bladder lavage requires considerable skill but in my experience it is not necessary, and it is a more satisfactory procedure to fill the bladder with I per cent: citrate solution and connect the catheter to an aseptic drainage bottle when the patient returns to the ward. If the operation has been properly carried out bleeding is moderate and clotting never occurs.

It is essential during the operation to maintain the patient's blood pressure at a reasonable level or shock may develop and the urinary secretion diminish. The patient must drink freely as his urine is the best form of bladder wash.

Critics of punch prostatectomy point out that complete removal is impossible and that recurrence or carcinoma may follow. This criticism is justifiable but the experienced operator can remove the whole prostate at one or two sittings. Recurrence of obstruction is unlikely except with very inadequate removal of large adenomatous glands. The fibrous type of gland does not cause recurring obstruction provided a moderate amount of tissue has been taken away. The main advantages of this operation in my experience are that very little shock is produced, good results can follow the removal of a small amount of ti:sue 
and the operation can be abandoned at any time. Chest complications are uncommon, post-operative discomfort trivial, and the patient is kept in bed for not more than 24 or 48 hours. It is, therefore, very suitable for the aged and feeble and these advantages are appreciated by the middle aged.

\section{Conclusions}

Before the merits of the various methods of treating prostatic obstruction are discussed, it is well to recall to mind the aims of treatment - the saving of life and the restoration of normal bladder function-and to remember that probably in no other field of surgery is the human material less robust.

Prostatic obstruction is a very common disease affecting old men when they should be expecting some peace and comfort. No practitioner is without experience of the condition, and no hospital without its beds and even wards for prostatics. Many operations have been devised for the treatment of this condition, some simple, many complex, and so long as general surgeons have to perform the majority of prostatectomies the choice of treatment must remain largely individual and simple methods must hold the field.

In considering the merits of any operation the number of cases rejected as unfit and condemned to permanent suprapubic cystotomy must be taken into account, also the morbidity associated with temporary drainage and the dangers of more than one operation must be remembered. It is probably true that the safest and simplest operation is suprapubic cystotomy of the Freyer-Fuller type after a period of temporary drainage, and I think this operation must remain the most widely useful; but it may be supplanted by Millin's prostatectomy which shows great promise. Wilson Hey's recent results are excellent but his operation has not yet been widely adopted, so a final verdict cannot be given. Transurethral prostatectomy, the most difficult of all is, in my opinion, the operation of the future for it is applicable in every case. It can be done in one or more stages, and it is performed by the masters with a mortality of less than I per cent. Although America is still the home of this operation, it is gaining ground in England as recent publications by Wardill and Stewart show.

All the methods described above give excellent results if carried out properly, and none is particularly prone to complications. Some require longer recumbency than others, some produce greater shock and are, therefore, not suitable for all comers. My preference is to perform prostatectomy with the cold punch in all cases except those with very lárge, soft adenomatous glands, and for these " $I$ find is Millin's operation the most satisfactory.

To conclude I will quote the opinion of the late Dr. Hugh Cabot, one of the greatest urologists :-

' The real nub of the question turns upon the decision as to who is to treat by operation these people with obstructing prostates. If they are to be treated in the future, as in the past, largely by the general and, perhaps, $\vec{\odot}$ occasional surgeon, then' the indication is for $ᄋ$ an operative method as nearly fool proof as may be. But this does not seem a very high $\frac{}{0}$ ideal for surgical practice . .

' It 'is, perhaps, safe to guess that supros $\vec{\circ}$ pubic prostatectomy will long hold a plae. where expert urologists are not available: Perineal prostatectomy will have to fight it ou with the transurethral resectionists. Both of the operations require prolonged training, $\frac{0}{\circ}$ great skill and dexterity, and large experience. Given these in equal measure, I suspect that the younger generation, armed with better equipment, will win the day for transurethral operations.'

\section{BIBLIOGRAPHY}

BUMPUS, HERMON C. (ro36), Cabot's " Motern Urology." CABOT, HUGH (1936), “Modern Urology.?" Henry'Kimpton. EMMETT, JOHN L. (1943), F. Urol., 49, 6.

FREYER (1901), B.M.ศ., ii, 125; lbid (1902), i, 249 and ii, 245; (1942), Ibid; (1903), i, 898

FULLER (1895), "Cutar and Genito-Urinary Disease," p.23.

HARRIS, S. H. (1928), "Prostatectomy with Complete Closure," F. Coll. Surgeons, Australasia, I, 65.

HARRIS, S. H. (1928), Med. F. Australia, 2, 288.

HEY, WILSON (1945), B. 7. Surg., 33, 129, 41.

KIRWIN, T. J. (1931), F. Surg. Gynec. and Obstet., 52, 1007. Oे

MCCARTHY, J. F. (193I), 7. Urol., 26, 695.

MCGILL, . (1888), Trans. Clin. Soc., London, xxi, 52.

MILLIN, TERENCE (1945), Lancet, Dec., I.

STERN, MAXIMILIAN (1926), Phys. Therapy, 44, 439.

STERN, MAXIMILIAN (1926), F.A.M.A., 87, 1726.

STEWART, H. H. (1945), B.M. .

WALKER, KENNETH M. (1923), “Diseases of Male Organs of Generation," Oxford Med. Publications, London.

THOMSON-WALKER, J. W. (1930), Tr. Med. Soc., London, 53, 143 .

WARDILL, W. E. M. (1941), Lancet, Aug., 2.

YOUNG,IHUGH HAMPDEN (1903), f.A.M.A., ii, 999. 